\title{
I. On the inorganic constituents of organic bodies
}

\section{H. Rose}

To cite this article: H. Rose (1849) I. On the inorganic constituents of organic bodies, Philosophical Magazine Series 3, 35:233, 1-24, DOI: 10.1080/14786444908646293

To link to this article: http://dx.doi.org/10.1080/14786444908646293

册 Published online: 30 Apr 2009.

Submit your article to this journal 준

Џ Article views: 2

Q View related articles $₫$ 
T H E

LONDON, EDINBURGH AND DUBLIN

PHILOSOPHICAL MAGAZINE

A N D

JOURNAL OF SCIENCE.

[TIIRD SERIES.]

$J U L Y$ 184.9.

I. On the Inorganic Constituents of Organic Bodies. By H. Rose, Professor of Chemistry in the University of Berlin *.

TTHE inorganic constituents of vegetable and animal sub1 stances have received more attention during the last few years than was formerly the case; and in consequence of Liebig's exertions especially, numerous investigations of the ashes of organic substances have been made; but their principal object was only technical. It was soon perceived, that as plants derive the inorganic constituents, without which they cannot exist, from the soil, it was of the greatest importance to determine these constituents with accuracy, so as to be enabled to judge whether the soil was capable of yielding them; and if not so, to allow of their being added in the form of a suitable manure.

Hitherto almost all these investigations have been directed to the determination of the relative proportions of the inorganic constituents of the ash. Occasionally, indeed, the presence of certain inorganic constituents, especially salts, has been detected by microscopic examinations; but scarcely any one has expressly taken up the question which is somewhat closely connected with this point, viz. in what manner are the inorganic substances combined with the organic? Whether they form with each other the same kinds of combinations as those which are artificially prepared in our laboratories, or whether peculiar compounds, existing only in living organic bodies, are formed by the mutual influence of the inorganic upon the organic matters, are questions which must be of great importance, especially in animal and vegetable physiology, and which have not hitherto been accurately investigated.

In very few instances only have researches of this kind been attenpted. Fndeavours have been made, for instance,

* From Poggendorf's Annalen, vol. Ixxvi. p. 305.

Phil. Mag. S. 3. Vol. 35. No. 233. July 1849. 
to show the manner in which the iron is combined with the other constituents of the blood. But these and some other experiments of the same kind form isolated examples; at least it has never been attempted to ascertain the changes which the inorganic constituents supplied to vegetable and animal bodies by the soil and articles of food undergo within them.

Some time since, I endeavoured to prove, that when an organic substance of vegetable or animal origin is carbonized with exclusion of the air, by not too great a heat, the inorganic constituents can partly be extracted by the ordinary solvents of inorganic salts-water and muriatic acid, but that a portion, and this frequently the largest, exists in the carbonized residue of some organic substances in such a state as completely to resist the solvent action of water and muriatic acid, and can only be obtained by burning the carbonized mass in oxygen gas or in atmospheric air*. This portion of the inorganic matters undoubtedly does not exist in the organic substance in the state in which it is obtained after the incineration, nor even in the carbonized mass obtained from it, but it has been formed by oxidation. I also described several experiments, which showed that the presence and the nature of the carbonized mass could not be the causes of the insolubility of the inorganic substances in the solvents, even if they pre-existed in the organic bodies in the same state as that in which we find them in the ash.

These remarks, which $I$ published in an imperfect state and supported by few proofs, for the sake of directing the attention of chemists to this, as it appeared to me, not unimportant subject, have not received any attention. Since that time, I have, however, occupied myself with these investigations, and have induced several young chemists in my laboratory to determine the inorganic constituents, with a view to separating those which exist already formed in organic bodies from those which must be contained in them in an unoxidized or less oxidized state.

These investigations have completely confirmed the view I had arrived at from reflecting upon this subject. In fact $I$ may assert, that in none of my chemical investigations has experiment so completely confirmed the hypotheses which I had made before the commencement of the undertaking than in these.

W'hen we minutely trace the entire process by which plants and animals assimilate the inorganic substances consumed, it appears that this is effected in the two cases in an entirely opposite manner. I shall now investigate this more minutely.

* Chem. Gaz., vol. v. p. 158. 


\section{The Inorganic Constituents of Plants.}

Plants obtain their inorganic constituents by means of the roots, which derive them from the soil upon. which they grow. The latter either already contains them among its constituents, or they are added to it in a suitable manure. In both cases these inorganic constituents exist in the highest possible state of oxidation. If they are not contained in the manure in this state, the latter is not in a perfectly proper condition, and does not become so until it has been exposed to the air for some time.

It may certainly be admitted, that the inorganic constituents, absorbed by the root in the most perfect state of solution possible, ascend in the vessels of the stem. Probably those salts which are insoluble in water are dissolved by the aid of carbonic acid.

During the growth of the plant a deoxidizing process goes on within it; the green parts under the influence of solar light, as is well known, evolve oxygen gas. Even when they only decompose the carbonic acid of the air, they assimilate its carbon; hence the amount of the latter in the plant gradually increases in proportion to that of the oxygen. All those parts of the plant which are in contact with the green parts take a share in this deoxidizing process, so long as it continues in a state of growth and the green parts have not lost their green colour. Now when we find that in plants one portion of the inorganic constituents absorbed by the root exists in a deoxidized state, in which, at least after the carbonization of the plant, it is insoluble in the ordinary solvents-water and muriatic acid, and that this portion is not converted into the same salts as those absorbed by the root from the soil until after oxidation, it may be supposed that the quantity of deoxidized inorganic constituents must be small in those parts of the plant which are in more immediate contact with the soil, and in which, therefore, the deoxidation of the parts of the plant, and also that of the inorganic salts existing in them, first commenced. But this quantity must be greater in those parts of the plant the formation of which has required the longest time; and after the formation of which, many plants, including all the annuals, die. Hence the proportion of the inorganic constituents of the plants which are deoxidized to that of those which are not deoxidized, must be very different in the herbaceous portions of the plant and the seeds.

This supposition has been most completely verified by experiment.

M. Weber made a comparative experiment upon the inorB 2 
ganic constituents of peas and pea-straw, which is more fully described in the appended supplement (I. and II.). They were both carbonized, with exclusion of the air, at such a temperature, that the water with which the carbonized mass was treated was not coloured yellow or brown, but remained perfectly colourless. Water extracted from the carbonized mass of the peas a large amount of chloride of potassium and phosphate of potash, some chloride of sodium and sulphate of potash, and a considerable quantity of carbonate of potash : the latter must have been contained in the peas, before carbonization, in the form of the potash-salt of an organic acid. Water extracted from the carbonized mass of the pea-straw a far larger amount of carbonate of potash, but smaller quantities of chloride of potassium, chloride of sodium, sulphate of potash, silicate of potash, and sulphate of lime.

When the carbonized mass, which had been exhausted with water, was then treated with muriatic acid, the latter dissolved out of the carbonized peas phosphate of soda, potash, lime, magnesia, and perphosphate of iron, but no earthy carbonates; whilst from the carbonized pea-straw this acid extracted a large quantity of carbonate of lime, a small quantity of carbonate of magnesia, and the phosphates of lime, magnesia and iron, but none of potash or soda.

The carbonized mass which had been exhausted with water and muriatic acid was burnt with access of air. The ash of the peas consisted of the phosphates of potash, lime, magnesia and iron; that of the pea-straw was composed of the phosphates of magnesia, lime and iron; it contained no potash, but a considerable quantity of silica.

On comparing the amounts of inorganic matters obtained from the peas and the pea-straw by these two operations, the following results are obtained. $100 \mathrm{grms}$. of peas and 100 grms. of pea-straw yielded-

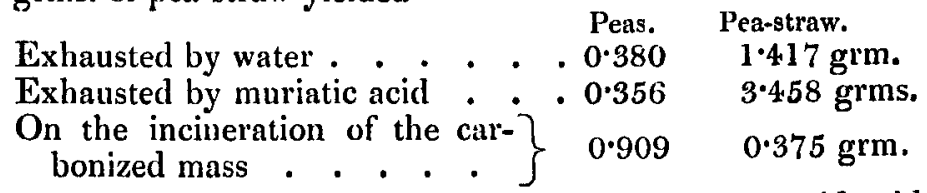

Hence water and muriatic acid dissolved very considerable quantities of inorganic constituents from the carbonized peastraw, but not more than a sixth or seventh of this amount from the peas; whilst on the incineration of the carbonized mass which had been exhausted by solvents, far more fixed salts were formed in the case of the peas than in that of the pea-straw.

The proportion in the latter case is, however, far more 
remarkable than is at first sight apparent; for the ash of the exhausted carbonized mass of the pea-straw contains more than half its weight of silica, which existed as such in the oxidized state in the living plant, and could not be obtained until after the incineration of the carbonized mass, merely in consequence of its insolubility in the solvents. If we take this circumstance into account, the exhausted carbonized mass of the peas yields five times as large an amount of fixed salts as that of the pea-straw.

Exactly the same results were obtained in the analysis of the inorganic constituents of rape and rape-straw, made by M. Weber (Appendix III. and IV.).

The quantities per cent. of inorganic constituents were-

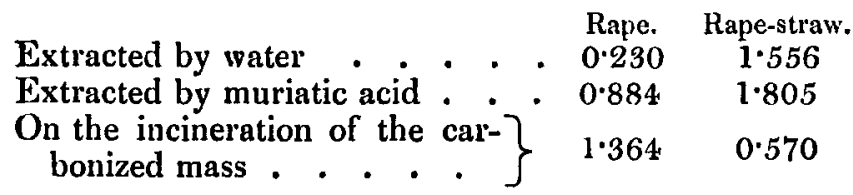

Here also the proportion is more striking than appears at first sight, because nearly half of the ash of the carbonized mass of the rape-straw after exhaustion with the solvents, consists of silica, as in the case of the pea-straw.

The salts obtained by the incineration of the carbonized mass after exhaustion by the solvents, could not have existed as such in the plants and seeds, as has been already mentioned, nor even in the carbonized mass itself, but must have been produced by oxidation. The next question then is, in what states of combination did the unoxidized inorganic constituents exist in the plant, and what in the carbonized mass obtained from it? Upon this point all our explanations are insufficient; and this question can only be answered by hypothesis, which cannot be verified withont further investigations. But at all events the subject forms a wide field for interesting investigations, which lead to important results, and by which both chemistry and physiology may acquire important elucidation.

The carbonized residue of peas and rape, after exhaustion with water and muriatic acid, yields on incineration a large amount of phosphates, and which, bad they existed as such in the carbonized vegetable matter, would have been dissolverl by the water and muriatic acid. The carbonized mass also contains considerable quantities of nitrogen. If the phosphorus existed in the unoxidized state, it is most probable that it formed compound radicals with carbon and nitrogen, similar to cyanogen or sulphocyanogen, which were combined with the 
metals of the basic oxides contained in the ash. Hence in proportion as the deoxidizing process proceeds in the living plant, the phosphates which are absorbed from the soil by the roots become converted into non-oxidized compounds, consisting of compound radicals containing phosphorus and the metals of the alkalies and earth. These must exist in the largest quantity in those parts of the plants which are composed of substances exposed for the greatest length of time to the deoxidizing process; and these are evidently the seeds of the plants, which are formed latest, and upon the production of which the life of many plants entirely ceases.

Thus the phosphates are decomposed in plants by the deoxidizing process in the same manner as the sulphates are converted into sulphurets by deoxidation. Probably the more appropriate explanation is, that when substances containing nitrogen and carbon are present, sulphocyanurets, or compounds of a radical consisting of three elements, sulphur, carbon and nitrogen, may be formed from the sulphates by a deoxidizing process.

If we admit the occurrence of these compounds of hypothetical radicals with metals in the seeds of plants, the next point, after the determination of their properties, would be the investigation of their relation to the great mass of the organic matter in those parts of the plants in which they exist. But as even the existence of these compounds is problematical, it would be useless to form further hypotheses on this point before making ourselves somewhat more intimately acquainted with these compounds themselves.

The so-called proteine compounds appear to be formed principally in plants by the process of deoxidation; and it is precisely these substances which appear to combine with the radicals containing phosphorus, in combination with metals.

Another question must be proposed here, although it cannot be satisfactorily answered. Supposing that these compounds really exist in certain parts of plants, what changes do they undergo when the plant is carbonized with exclusion of air; when consequently all the organic matter is destroyed, and the connexion in which they probably stood to these compounds is dissolved? So long as we have no certain knowledge of the existence of these compounds, we cannot judge with certainty of the changes which they experience at an elevated temperature. It is however possible, at least not improbable, that they may be acted upon at an elevated but not too high a temperature in the same manner as the cyanurets, when they are transformed by heat into paracyanurets. Be this as it may, the experiments show that these compounds 
after carbonization are insoluble both in water and in muriatic acid. But this insolubility in the solvents did not always exist before carbonization; for many organic substances, which in an undecomposed and not carbonized state are soluble in water, frequently contain a large part of their inorganic constituents in an unoxidized state.

It is well known, that most of, but not all, those inorganic salts which are insoluble in water are soluble in muriatic acid. But those salts which are insoluble in this acid certainly very rarely occur in vegetable and animal substances. The metaphosphates require most attention in this respect, since they may exist in the carbonized substance; and yet, on account of their insolubility in water and muriatic acid, their presence may only be detected after the destruction of the carbonized mass. But it is evident, from the investigations which have been made upon the inorganic constituents of vegetable and animal substances, and which are described in the Appendix, that these metaphosphates can rarely if ever be present, since in most cases carbonate of potash may be removed from the carbonized mass by water, and which, as is well known, cannot exist simultaneously with metaphosphates at an elevated temperature.

We must next mention the remarkable occurrence of silica in vegetables, principally in the stems of the Grasses and Equisetacee (Appendix V.). The silica is undoubtedly removed from the soil by the plants in the form of silicate. It becomes separated, however, from this silicate ; and the silica separated forms the principal mass of the stem in several species of $E q u i$ setum and the Grasses. Of course it exists in them in the form of perfectly oxidized silicic acid ; but in consequence of its insolubility in water and muriatic acid, the greater part of it is found in the mass remaining after the exhaustion of the carbonized substance with water and acid.

Hence on comparing the quantities of the inorganic substances which the seeds and the culm of grasses yield in the aqueous and muriatic solutions of the carbonized substance, and on the combustion of the latter, we find, in contradiction to the above determination, that the carbonized straw yields far more fixed substances than the seeds. But this contradiction is only apparent, because the substances obtained after the incineration of the carbonized straw consist almost entirely of silicic acid.

A comparative examination of the inorganic constituents of wheat and wheat-straw made by $\mathrm{M}$. Weber, and which is fully described in the Appendix (VI. and VII.), proves this beyond a doubt. 
Water extracted chloride of sodium and phosphate of potash and soda from the carbonized grains of wheat, but no carbonates; whilst from the carbonized wheat-straw it removed chloride of potassium, chloride of sodium, some sulphate of potash, and no phosphates, but a remarkably large amount of silica. Muriatic acid subsequently dissolved only compounds of phosphoric acid with potash, soda, lime, magnesia and peroxide of iron. From the carbonized straw, muriatic acid extracted combinations of phosphoric acid with lime, magnesia and peroxide of iron, as also some silica.

The carbonized grains of wheat, after exhaustion by the solvents, yielded on combustion, compounds of phosphoric acid with potash, lime, magnesia and peroxide of iron; also some silica. The solids remaining after the combustion of the carbonized wheat-straw consisted almost entirely of silica, with extremely small quantities of the phosphates of lime, magnesia and peroxide of iron.

On comparing the amounts of the constituents obtained in the different operations, we obtain the following results. 100 grms. of the grains of wheat and of the straw yielded-

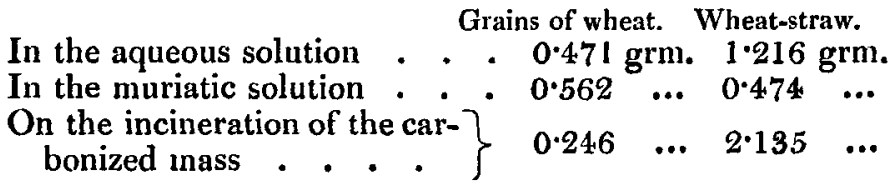

Of the $2 \cdot 135$ grms. of fixed constituents which the carbonized wheat-straw yielded, 2.022 grms. consisted of silica, and only $0.113 \mathrm{grm}$. of phosphates. When this is taken into consideration, this investigation also proves that the quantity of so-called deoxidized inorganic constituents in the grains of wheat is greater than that in the wheat-straw.

Perhaps it is of importance to distinguish the organic substances, the inorganic constituents of which are contained in them in a completely oxidized or deoxidized state, by separate names.

I sball therefore call those urganic substances, the inorganic constituents of which are in a perfectly oxidized state, teleoxidic bodies. Pea-straw and rape-straw, as also even wheat-straw, may be called teleoxidic substances, although they do not deserve this appellation so strictly as many animal bodies, of which we shall speak presently. But those organic substances, the inorganic constituents of which exist partly in an oxidized and partly in an unoxidized state, I shall call meroxidic sub- 
stances. Peas, rape and wheat, are therefore meroxidic substances. I have never met with perfectly anoxidic substances, either in the examination of vegetable or animal substances. It is probable that if the proteine compounds existing in meroxidic substances were isolated as completely as possible, they would constitute perfectly anoxidic substances, which after carbonization would not yield any soluble salts to the solvents, until these had been produced by complete incineration.

\section{Inorganic Constituents of Animals.}

Animals derive the inorganic constituents which the various parts of their body contain, from the food. This is, however, assimilated by them in a totally different manner to that in plants; whilst in the latter, in general, a process of deoxidation occurs, to which the inorganic substances derived from the soil are subjected, in animals the nutritive substances undergo oxidation by the oxygen inspired. They are first converted into blood, and this is conveyed to all parts of the body, where reparation must occur. By the oxidation of the nutritive substances, or rather of the matters produced from them, the elevated temperature of the aninal body is produced; and as this is tolerably uniform, the oxidation also must go on equally uniform in the various parts of the body. It is not, however, merely those parts of the body which consist of carbon, hydrogen, nitrogen and oxygen only, that take part in the oxidation, but undonbtedly also those compounds of the hypothetical phosphuretted radicals with metals, which such animals as are not carnivorous derive from the meroxidic substances of vegetable nutritive natters. 'That portion of them which is not applied to the repair of the body becomes oxidized; the same also occurs with those parts of the body which are repaired. Whilst the carbonic acid of these compounds is expired in the form of carbonic acid, and the nitrogen is converted into ammonia, the phosphorus is oxidized to form phosphoric acid, and the metals combined with the radicals, so as to form oxides. The longer these substances have been exposed to oxidation, the more perfectly are phosphates of the metallic oxides formed.

It must follow from this conclusion, that the matter first formed by the nutritive substances, the blood, from which the other parts of the body are repaired, may contain completely oxidized salts, since it is generated from meroxidic substances, but must still contain a large amount of the combinations of the hypothetical radicals with metals. Somewhat the same must occur with flesh, the composition of which is the same 
as that of the blood; but as it is formed from the blood, it must contain more oxidized inorganic salts and less deoxidized salts than the blood. But if oxidation still continues, the inorganic constituents, which become perfectly oxidized by the inspired oxygen, must finally be removed from the body, as they are of no further use in it. Thus we find, in fact, that the inorganic constituents of the fluid and solid excrementitious matters are in a perfectly oxidized state, and are perfectly teleoxidic substances. Experiments upon the inorganic constituents of the blood, flesh, and the excrements, have completely confirmed these suppositions.

The inorganic constituents of the blood of the ox have been examined by MM. Weber and Merk (Appendix VIII.).

In the blood which was used for this purpose, the clot certainly had coagulated from the liquor sanguinis; but they were both carbonized without separation.

Water extracted from the carbonized mass a very large amount of chloride of sodium, carbonate of potash and of soda, and mere traces of phosphate and sulphate of potash.

Muriatic acid removed from it an inconsiderable amount of the phosphates of soda, potash, lime, magnesia, and perphosphate of iron.

The carbonized mass after exhaustion with these solvents yielded, on incineration, the phosphates of soda, potash, lime, and magnesia, with a large quantity of peroxide of iron and a small quantity of silica; it also contained traces of sulphates.

The following are the relative amounts of the inorganic constituents obtained by the three operations :-

In the aqueous solution . . . . . . 3.920 grms.

In the muriatic solution . . . . . . $0.389 \quad \ldots$

$\left.\begin{array}{c}\text { On the incineration of the remainder of } \\ \text { the carbonized mass . . . . . }\end{array}\right\} 2 \cdot 128 \quad \ldots$

We thus see that the amount of oxidized salts in the blood is very large. 'They consist, however, for the most part of salts soluble in water; and of these chloride of sodium, which cannot be considered as an oxidized salt, is the principal constituent, forming more than half of the whole amount of these salts. When this is taken into consideration, the quantity of inorganic constituents formed by the incineration of the carbonaceous mass after exhaustion with the solvents is larger than that of the salts contained in the blood. Hence the blood is a meroxidic body.

Flesh (that of the horse) has been examined as to its inorganic constituents by M. Weber (Appendix IX.).

Water extracted from the carbonized flesh small quantities 
only of metallic chlorides, and of sulphate of potash, a large quantity of alkaline phosphates, but no alkaline carbonate.

Muriatic acid then dissolved a considerable quantity of phosphates from the mass.

The residual carbonaceous mass yielded an ash which also consisted of phosphates.

The following were the relative amounts of inorganic constituents obtained in the analysis:-

In the aqueous solution . . . . . 3.090 grms.

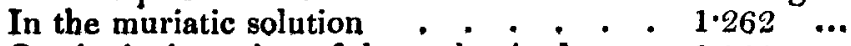

On the incineration of the carbonized mass $2 \cdot 866 \quad \ldots$

Hence flesh, like blood, is a meroxidic substance. The latter apparently contains a comparatively larger amount of teleoxidic matter than flesh. But the aqueous solution of the carbonized blood contains nearly 60 per cent. of chloride of sodium, whilst that of the carbonized flesh contained only very small quantities of alkaline chlorides. On taking this into consideration, the quantity of anoxidic matter in the blood is larger than in the flesh, and that of the teleoxidic matter smaller in the blood than in flesh.

The inorganic constituents of the liquid and solid excrementitious substances have been determined by M. Fleitmann (Appendix X. and XI.).

The extraordinarily large amount of salts contained in the urine is well known : they exist in it in a perfectly oxidized state. On evaporating the urine and carbonizing the dry residue, with exclusion of the air, water extracts almost the whole of the salts. On treating the carbonized mass, after exhaustion with water, with muriatic acid, a somewhat considerable amount of phosphates is further dissolved, part of which had been separnted by the evaporation of the urine.

The carbonized mass remaining after the action of water and muriatic acid, yielded so small a quantity of ash on combustion, as to give rise to the supposition that the inorganic constituents obtained in this way had also existed in an oxidized state in the urine, and had merely escaped the action of the solvents in exhausting the carbonized mass. One of the principal constituents of these inorganic substances was silica, which must have been separated on the evaporation of the urine and heating the dry residue, and have thus become insoluble in muriatic acid. The urine may therefore be considered as one of the perfectly teleoxidic substances.

The quantities of the inorganic constituents obtained in these operations were as follows:- 
In the aqueous solution . . . . . 54.148 grms.

In the muriatic solution . . . . . . $5.085 \quad \ldots$

On the incineration of the carbonized mass $0.352 \quad \ldots$

From the carbonized fæces water extracted some chloride of sodium and potassium, a considerable quantity of carbonate and phosphate of potash, a less amount of sulphate of potash, and a very large quantity of free potash.

But the quantity of inorganic constituents dissolved by muriatic acid was very large. It consisted of much phosphate of lime and magnesia, phosphate of potash and soda, a very small amount of sulphate, silicate of potash, and a very small quantity of peroxide of iron.

The exhausted carbonized mass on incineration yielded no very inconsiderable quantities of fixed constituents, the principal of which, however, was silica in the form of sand (san.. dartige Kieselsäure). It moreover contained a tolerably large amount of lime and magnesia, with a small quantity of phosphate of potash and soda, peroxide of iron and silica.

The relative quantities in the different solutions and in the remaining carbonized mass were as follows:-

In the aqueous solution . . . . . 1.933 grms.

In the muriatic solution . . . . . $6.493 \ldots$

On incineration of the carbonized mass $1.996 \quad \ldots$

But on deducting from the latter amount that of the silica and sand, we only obtain somewhat more than 1 grm., and hence the solid excrements may be considered as belonging to the teleoxidic substances.

Whilst those inorganic oxidized constituents which are soluble in water are separated by the urine, those which are insoluble pass off as fæces. In proportion as digestion in the body goes on in a normal state, and the less the excess of nutritive substances introduced into it, the amount of imperfectly oxidized constituents of the fæces must be less, and the quantity of teleoxidic substances larger. It is therefore probable that, by the chemical investigation of the fæces, a conclusion may be formed as to the proper or defective manner in which digestion is carried on. The fact that the inorganic constituents of the liquid and solid excrements, especially the phosphates, exist in them in the oxidized state, is the principal reason of their use as manure, which must be more fit for this purpose in proportion to the amount of teleoxidic substances of which it consists.

As the inorganic substances from animal bodies are added to plants in the form of manure, the process, in which they are first deoxidized and then again oxidized, recommences. 
The examination of other parts of the animal body by the method described is of some interest. As regards the bones, it is well known that all their inorganic constituents may be extracted by dilute muriatic acid, so that the cartilage remains in a pure state: likewise when they have been heated to redness with exclusion of the air, muriatic acid dissolves the inorganic salts, and leaves pure carbon. We know that bone-black, as it is called, may be decomposed in this manner.

Thus the bones, like the liquid and solid excrementitious matters, contain the larger amount of their inorganic salts in a perfectly oxidized state, and entirely belong to the teleoxidic substances. 'This explains the fact that ground bones form one of the best manures.

The bile (ox-bile) has been examined by M. Weidenbusch in the same way (Appendix XII.). Likewise, in this case, the surprising result has been arrived at, that the inorganic constituents, as in the excrements, exist almost wholly in the oxidizerl state, and that the bile is therefore a teleoxidic substance.

By far the larger portion of the inorganic constituents of the carbonized mass of the bile is extracted by water. It dissolves principally the carbonate, phosphate, and sulphate of soda, with chloride of sodinm; the quantity of salts of potash dissolved is, however, very small.

Muriatic acid extracted from it a small quantity of phosphate and a trace of silicate. The bases were principally lime, soda, potash, magnesia and the protoxides of iron and manganese.

The washed residue yielded a very small quantity of ash on incineration, which contained a remarkably large amount of sulphuric acid, and but little phosphates. The bases were principally soda, potash, magnesia and lime.

The following are the quantities of the inorganic constituents which were obtained in the various operations :-

In the aqueous solution . . . . . . 16.018 grms.

In the muriatic solution . . . . . . . . $0.869 \quad \ldots$ Un the incineration of the carbonized residue $0.7445 \ldots$

It is thus evident that the inorganic constituents of the bile exist in an almost complete state of oxidation, precisely as in the urine.

The next question is whether the milk is also an excretion, like the urine, and whether the whole of its inorganic constituents exist in a perfoclly oxidized state. It appears, however, from M. Weber's experiments upon the milk of the cow, that in addition to numerous perfectly oxidized salts, it also contains a considerable amount of inorganic constituents in a 
deoxidized state, and is therefore a meroxidic substance (Appendix XIII.).

The aqueous extract of the evaporated and carbonized milk contains a large amount of alkaline chlorides, with phosphate and carbonate of potash.

The muriatic extract of the carbonized mass contains a large amount of phosphate of lime, with small quantities of the phosphates of magnesia, potash and soda.

On the incineration of the exhausted carbonized mass, further large quantities of the phosphates of lime, potash, soda and magnesia were obtained.

The following are the relative proportions of the inorganic constituents obtained in the various operations:-

In the aqueous solution . . . . $7 \cdot 125$ grms.

In the muriatic solution . . . . . $6.621 \quad \ldots$

On the incineration of the remainder of

the carbonized mass. . . . . . . $\} 7^{\cdot 109} \quad \ldots$

Thus the milk contains a considerable amount of both oxidized and unoxidized inorganic constituents. It cannot under any circumstances be arranged in the same class as the urine, and like the latter, be considered as an excretion. It is a meroxidic compound.

In eggs (hens' eggs) the inorganic constiruents are in a more oxidized state in the albumen, whilst they are in a more unoxidized state in the yolk, as appears from the experiments of M. Poleck (Appendix XIV. and XV.). These experiments, however, were made long ago in my laboratory; the methods adopted were consequently imperfect, and they require repetition. This is the more necessary, since the results of these experiments appear to be in opposition to those which have been obtained in almost all the others. For all those meroxidic substances which have been examined, such as peas, rape-seed and wheat, moreover the blood, flesh and the milk, contain a large amount of the so-called proteine compounds. In teleoxidic substances, the excrements and the bile, the proteine compounds are entirely absent; and in the straw of peas, rape and wheat, they exist in small quantity only, corresponding to the amount of meroxidic substances found in them. It is probable therefore that the proteine compounds, when freed as perfectly as possible from all the teleoxidic substances which accompany them, are in many cases perfectly anoxidic substances. 'The only results opposed to this view, are those obtained by $M$. Poleck, as regards the albumen of eggs, which contains a small quantity of anoxidic substances. 


\section{Method of examining the Inorganic Constituents of Organic Substances.}

Vegetable substances are first freed as completely as possible from all foreign admixtures; solid animal matters are exposed to a gentle heat, to remove the greater part of the water they contain; and animal liquids are to be evaporated to dryness at a gentle heat. In this state the substances are placed in a Hessian crucible, the lid of which is furnished with a hole in the middle, the joints between the lid and the crucible are carefully luted with clay, and the crucible then placed for a long time in a hot place, and, if possible, all the remaining moisture expelled. It is then exposed to a moderately strong heat in a wind-furnace in a charcoal fire; the gases which escape through the hole are inflamed, and when the flame has burnt out and no more gases are evolved, the hole is closed with an accurate stopper of chalk, the crucible heated at a very low red heat, and then allowed to cool with perfect exclusion of the air.

The examination of the carbonized mass resolves itself into three parts.

Part 1.-The carbonized mass is first powdered as finely as possible, then boiled for a long time with water in a platinum dish, filtered and washed with hot water until a few drops of the filtering liquid leave a slight residue only, when evaporated upon platinum-foil. It is scarcely possible to continue washing the mass until the liquid which runs through leaves no residue, because a little phosphate of lime is always dissolved. It is therefore best, when the residue obtained on evaporating the water used in washing is very slight, to test a few drops of the water which runs through with solution of nitrate of silver; if a slight opacity only is produced, which disappears on the addition of nitric acid, this arises from phosphate of lime in solution, and we may be certain that all those constituents which are soluble in water have been removed. If the turbidity does not disappear on the addition of nitric acid, this arises from the water used in washing still containing some chlorides, and the washing must be continued longer.

On boiling the carbonized organic substances with water, as also on evaporating the aqueous extract, I have never been able to detect the evolution of ammonia. Hence, during the carbonization, neither alkaline cyanides nor alkaline cyanates are formed.

In all those substances which have hitherto been examined, the aqueous solution contained chlorides. If the amount present is small, the carbonized mass is easily washed; but if they 
form a principal constituent, the carbonized mass frequently requires to be washed for several weeks.

It sometimes happens that the aqueous solution appears of a brownish colour, which depends upon the imperfectcarbonization of the substance. Usually the liquid becomes decolorized on concentration, and deposits woolly flakes of carbon, which may be easily separated by filtration, before other substances are deposited from it.

If alkaline carbonates exist in the aqueous solution in any considerable quantity, free alkali is formed by the action of the carbon upon the alkaline carbonate, with the evolution of carbonic oxide. If the aqueous solution be evaporated, the dry mass contains more or less hydrated alkali with alkaline carbonate. By means of a solution of nitrate of silver, we may readily convince ourselves of the decomposition of the carbonic acid in the alkaline carbonates by carbon, since, unless the mixture has been exposed to a very great heat, this reagent does not produce a pure white precipitate in the filtered aqueous solution, but a more or less brownish precipitate is formed, which contains oxide of silver as well as the white carbonate of silver.

Decomposition of the alkaline carbonates ensues principally when the organic substance during its carbonization evolves a very large quantity of olefiant gas. Hence it takes place to a great extent when such seeds as contain a large amount of a fatty oil are carbonized, as rape-seed; it occurs to a much less extent on the carbonization of other seeds which do not contain any remarkable quantity of fatty oil, as peas, and likewise with the herbaceous parts of plants.

Unfortunately my attention was not directed to this circumstance until all the experiments described in the Appendix were entirely or partly finished. The alkali among the constituents of the aqueous extract is therefore assumed as existing in the state of hydrate.

It is therefore advisable to pass a current of carbonic acid gas through the aqueous solution before evaporating it, so as to convert the bydrated alkali into the state of alkaline carbonate.

The aqueous solution is then evaporated to dryness in a platinum capsule. It usually happens that when the liquid is very concentrated, it becomes more or less turbid from the separation of phosphate of lime which was in solution. When the liquid has been evaporated to a small volume, the phosphate of lime which has separated may be removed by filtration; it is however difficult to free the aqueous solution from it perfectly in this way; in fact in some cases it cannot 
be done, especially when it contains a large amount of silica, which also separates on the concentration of the liquid. It is therefore best to evaporate the whole of the liquid to dryness, and to separate the phosphate of lime in the course of the analysis.

The residue is moderately heated until its weight remains constant. If it be heated too strongly, decomposition of the carbonates contained in it may readily occur, as when silica and phosphates are present carbonic acid is expelled, which in fact partly occurs, according to Heintz*, during evaporation. After determining the weight of the dry residue of the aqueous solution, it is analysed as follows :-

The quantity of carbonic acid is first estimated by means of pure nitric acid in a suitable apparatus. This is obtained by the loss in weight of the apparatus. If, during this operation, the silica has become separated in the acid liquid, it is removed by filtration, and the chlorine precipitated from the filtered liquid by solution of nitrate of silver. The excess of silver is removed from the liquid after the separation of the chloride of silver by muriatic acid, and it is then evaporated to dryness in a porcelain capsule. The dry residue is moistened with muriatic acid and treated with water. Some silica usually remains undissolved, which, when added to that previously obtained, gives the whole amount contained in the aqueous solution.

The liquid separated from the silica is treated with ammonia, by which the entire amount which has been taken up by the water is precipitated. It is filtered, washed, dried, heated to redness, and weighed. Its weight, when deducted from that of the residue after evaporation to dryness, yields the true weight of the aqueous extract. It is mixed with the carbonized mass which has been exhausted by water.

The liquid is then treated with oxalic acid. In very few cases only is a precipitate of oxalate of lime thrown down, in most cases it is not so. The oxalate of lime is determined as carbonate by the ordinary method.

The filtered liquid is then treated with a solution of chloride of barium. The precipitate consists of phosphate, sulphate and oxalate of baryta. It is filtered and washed. Although the oxalate of baryta is not very difficultly soluble, yet after a little practice we can readily judge when the washing must be discontinued. It is therefore advisable, on precipitating the lime, not to add too large a quantity of oxalic acid, so as to avoid obtaining a too copious precipitate of oxalate of baryta.

The precipitate is treated with dilute muriatic acid; sulphate

* Poggendorff's Annalen, vol. Ixxii. p. 120.

Phil. Mag. S. 3. Vol. 35. No. 239. July 1849. 
of baryta is then left undissolved, and its weight must be determined after it has been washed. We thus estimate the amount of sulphuric acid. The muriatic solution of the barytic salt is treated with sulphuric acid, and the precipitated sulphate of baryta separated by filtration. It is then supersaturated with ammonia and muriate of anmonia, and sulphate of magnesia added to precipitate the phosphoric acid. Its weight is calculated from the phosphate of magnesia, after having been heated to redness.

The liquid from which the insoluble barytic salts have been separated by chloride of barium, still remains to be examined. The excess of chloride of barium is decomposed by carbonate of ammonia. If the liquid is very dilute, it must be previously concentrated by evaporation. The carbonate of baryta is separated by filtration, the filtered liquid evaporated to dryness, and the ary residue carefully heated to redness to expel the ammoniacal salts. The remaining mass consists entirely of alkaline chlorides. 'These are separated in the ordinary way by chloride of platinum. The amount of potash is determined from the weight of the ammonio-chloride of platinum. The amount of chloride of sodium may be calculated from the loss; but it is better to determine it directly as sulphate of soda.

Par'T II.-This part of the investigation is commenced by boiling the carbonized mass, which has been exhausted by water, with muriatic acid for a long time; it is then filtered and washed with hot water, to which a little muriatic acid has been added, until a few drops of the water used in washing leave no residue when evaporated upon platinum foil. Thefiltered liquid is evaporated nearly to dryness in a platinum capsule. The weight of the dry residue cannot be determined, on account of the readiness with which the chlorides of iron and magnesium are decomposed by heat. The alkalies usually existed in the dry residue as chlorides, whilst they were contained in the exhausted carbonized mass in the form of phosphates; for when the carbonized mass has been washed with water, and a portion of the latter is treated with nitric acid, no precipitate is produced in this solution by solution of nitrate of silver. In arranging the constituents of the muriatic solution, their sum must be taken, and the alkalies represented in the state of oxides.

I was at first much astonished at finding alkalies present in the muriatic solution, as I had no reason to believe they existed in it. Subsequent investigations, however, which I shall describe in a future paper, have shown that when pyrophosphate of lime and pyrophosphate of magnesia are heated with not too large a proportion of alkaline carbonates, remarkable double 
salts, consisting of the earthy phosphates in combination with the alkalies, are formed. Most of them are analogous in composition to the ammonio-phosphate of magnesia, but contain a fixed alkali instead of ammonia. The extremely imperfect decomposition which ensues on fusing the phosphates of lime and magnesia with alkaline carbonates, depends in most cases upon the formation of these double salts.

Their formation is undoubtedly the cause of the quantities of the alkalies being estimated incorrectly, and in too small proportion, in many investigations on the determination of the inorganic constituents of organic substances, because their presence in the muriatic solution could not have been expected.

The mass obtained by evaporation is moistened with muriatic acid and then treated with water. Usually a small quantity of silica remains undissolved, which is separated by filtration and its weight determined. The liquid is then nentralized with ammonia. The precipitate contains phosphoric acid in combination with lime, magnesia and peroxide of iron. It is well known that when phosphate of magnesia has been heated to redness, and is then dissolved in an acid, it cannot be completely precipitated by ammonia : the error, however, arising from this source is of only slight importance; because the carbonized mass has been heated for a very long time with muriatic acid, by which means, as is well known, the pyrophosphate of magnesia is almost entirely converted into the $c$-phosphate of magnesia. The extremely small quantity of magnesia which remains in solution is, however, determined in the further progress of the analysis.

The phosphates which have been precipitated by the annmonia are dissolved in nitric acid, and treated with mercury to separate the phosphoric acid from the bases. The dried mass is treated with water, and the mercurial salt removed from the solution by muriate of ammonia and ammonia; the lime is then precipitated by an alkaline oxalate, and the magnesia by phosphate of soda. When peroxide of iron is mixed with the earths, it is separated by the usual methods. The insoluble mercurial residue containing phosphoric acid is fused with carbonate of soda, and the fused mass treated with water. Should any peroxide of iron then remain undissolved, it is separated by filtration, dissolved in muriatic acid, and precipitated by ammonia. The liquid which has been separated from the peroxide of iron by filtration, and which contains phosphate and carbonate of soda, is supersaturated with muriatic acid and then with ammonia, and the phosphoric acid precipitated by sulphate of magnesia. The amount of phosphoric acid is calculated from the weight of the am- 
monio-phosphate of magnesia after it has been heated to redness.

By the analysis of the phosphates precipitated by ammonia, the composition of the precipitated phosphate of lime and the presence or absence of the perphosphate or pure peroxide of iron in the precipitate may be determined. The liquid filtered from the earthy phosphates contains either alkalies and phosphoric acid only, as is the case in the examination of all seeds and animal matters, or it contains lime and magnesia only, and these frequently in very considerable quantities. They existed in the carbonized mass in the form of carbonates; but it is only in those cases in which they are present in considerable quantities, as in the analyses of the straw of peas and rape, that a very distinct evolution of carbonic acid can be perceived on treating the carbonized mass, after exhaustion with water, with muriatic acid. As the carbonic acid cannot be determined directly, it is calculated from the quantity of lime and magnesia found in the liquid filtered from the earthy phosphates.

In the analysis of the carbonized mass of vegetable seeds and animal substances, where, as has been previously mentioned, the liquid filtered from the earthy phosphates contains both alkalies and phosphoric acid, this is treated with chloride of barium; phosphate and, when sulphuric acid is present, sulphate of baryta are then precipitated. Sulphuric acid has, however, very rarely been found in the muriatic extract of the carbonized mass. The phosphate of baryta is separated by filtration, dissolved in muriatic acid, the baryta precipitated from the solution by sulphuric acid, which is then supersaturated with ammonia, and the phosphoric acid precipitated as ammonio-phosphate of ningnesia.

The liquid which was separated from the phosphate of baryta contains the alkalies. The excess of baryta is removed by carbonate of ammonia, the filtered solution evaporated to dryness, and the dry mass heated to redness to expel the ammoniacal salts. The alkaline chlorides left are separated by chloride of platinum.

The examination of the second portion can be considerably simplified when sulphuric acid is absent. The muriatic extract is carefully evaporated. If it contains silica, the dry mass is moistened with nitric acid, and the silica separated; mercury and nitric acid are adcled to the filtered liquid; it is then evaporated to dryness with excess of mercury in the ordinary way, and the bases separated from the phosphoric acid by water.

This simplified method has, however, two disadvantages. 
The large amount of chlorine present produces a large quantity of protochloride of mercury, which remains mixed with the protophosphate of mercury; hence this insoluble mixture requires a large quantity of carbonate of soda for its decomposition. The second disadvantage is, that when the earths form the principal constituents of the acid extract, it cannot be accurately determined whether they are combined with phosphoric or carbonic acid.

PAnT III.- This part of the investigation comprises the determination of the inorganic constituents existing in the car. bonized mass after exhaustion with water and muriatic acid, or rather which are produced by oxidation.

This incineration of the carbonaceous mass is accompanied by no small difficulties. I formerly effected it by heating it to redness in a crucible, the lid of which was perforated, and a silver tube, which conducted a current of oxygen to the heated mass, was fitted to the aperture. The incineration succeeded perfectly in this manner; but in a very large number of cases it was impossible to incinerate the carbonized mass merely by the access of atmospheric air without the aid of oxygen. But on combustion in oxygen gas, an elevated temperature is produced and the substance of the crucible is powerfully attacked by the phosphates formed by the oxidation. If a platinum crucible be used, it is completely spoiled when alkaline phosphates and carbon act upon it at an elevated temperature. Nor can a porcelain crucible be used; because when the greater portion of the carbon has been burnt, the alkaline phosphates, being in a state of fusion, dissolve the glaze. This is particularly the case with porcelain crucibles of Meissen manufacture; but even those manufactured at Berlin cannot resist the action, although they are far less acted upon than the former. Silver crucibles do not stand the high temperature, and begin to melt. When the combustion of the carbonized mass is effected in a glass tube heated to redness in a furnace by a charcoal fire, it succeeds tolerably well; but the high temperature produced by the combustion of the carbon in oxygen fuses the alkaline phosphates, and they unite with the glass, so that they cannot afterwards be separated from it mechanically. The combustion was finally effected in small earthenware crucibles, which answered in every respect tolerably well, and were but slightly acted upon by the alkaline phosphates; but one important difficulty still remained unconquered, - a considerable quantity of alkaline phosphate is constantly volatilized. This is very large; and the volatilization cannot in any way be avoided when the combustion is effected in oxygen gas, even when the current is slow. 
When the gas was passed through too slowly, combustion did not take place; but as soon as it was allowed to flow more quickly, the oxidation of the carbon occurred with the production of a vivid light, and white fumes were distinctly seen to ascend. For the purpose of examining the volatilized products, the combustion of the carbonized mass was effected in a tubulated glass retort, through the tubulure of which the oxygen was passed, whilst its mouth was immersed in water. The water was found to exhibit powerful reactions of phosphoric acid and alkali.

If a mixture of alkaline phosphate with a large proportion of carbon is artificially prepared, and the mixture burnt in a current of oxygen, the same phænomena as those just described are found to occur, and dense white fumes are seen to ascend.

This volatilization only occurs with alkaline phosphates. On the combustion of a carbonized substance, which after incineration yields earthy phosphates only, volatilization cannot be perceived. Moreover, when earthy phosphates are artificially mixed with carbon, and the mixture burnt in oxygen gas, volatilization does not occur, nor can any white fume be perceived. Hence the earlier experiments which were performed in my laboratory, in which the washed carbonized mass was burnt in oxygen gas, have yielded incorrect results, and must therefore be repeated, as a method has at last fortunately been discovered by which the combustion can be effected without loss. This method, which was proposed by M. Fleitmann, is as follows:-

The carbonaceous mass, after having been exhausted with water and muriatic acid, is dried, and then moistened with a concentrated solution of chloride of platinum. The moist mass is heated in a porcelain crucible or a porcelain capsule, at first gently, so that no moisture may be expelled and no loss occasioned by the spirting of the mass; then more strongly, so that it attains an incipient red heat. This is best effected in a large concave lid of a platinum crucible. If the whole be heated in a porcelain crucible, the incineration requires a far longer time, inasmuch as it takes place more slowly in proportion to the smallness of the surface and the less free contact of the air. A very slow combustion of the carbon ensues, chlorine being simultaneously developed; each carbonaceous particle becomes incandescent, just as in the scintillation of an inflamed vegetable substance. The conbustion is extraordinarily increased by constantly stirring the mass with a platinum wire or a small platinum spatula. As fresh portions of the unburnt carbon come into contact with the air, the scintillation recommences. The spirit-lamp may be removed, 
yet the carbon continues to glow for a long time; frequently, when the quantities are not too small, for several hours; even portions taken out with the platinum spatula continue to burn. The more concentrated the solution of platinum is, the more readily does the combustion ensue. When the mass ceases to glow on the continued application of heat, and still appears black, it must be again moistened with chloride of platinum. The less carbon the residue contains, the more readily does the combustion ensue. If the solution of platinum is concentrated, twice moistening and heating are usually sufficient to produce the combustion of the whole of the carbon. A second moistening with the solution of platinum is not strictly requisite. After the mass has been once moistened and then heated to redness, the residue may be treated with aqua regia and evaporated, so as to render the platinum again active; however, this is a somewhat tedious proceeding; great care must also be taken that no loss is occasioned by the spirting of the mass. As the whole of the platinum used is usually recovered, the method does not entail any pecuniary loss; it is therefore preferable to moisten the mass a second time with chloride of platinum.

When all the carbon has been burnt, the residuary mass is of a pure ash-gray colour. Before it is treated with muriatic acid, it must be heated to redness in a porcelain crucible in a current of hydrogen gas, so that the double compounds of the alkaline chlorides with the chloride of platinum may be completely decomposed, which can only be accomplished with difficulty with large quantities by merely heating them in atmospheric air. If the treatment with hydrogen gas be omitted, the subsequent separation of the platinum from the muriatic solution of the ash is accompanied with several inconveniences. The mass, after having been treated with hydrogen gas, is digested for a long time with muriatic acid in a flask, the residue separated by filtration, and washed with water containing muriatic acid. The resulting solution, which usually contains the same constituents as the muriatic extract of the carbonized mass, is analysed in exactly the same manner.

The platinum remaining undissolved, which is also mixed with the sand and silica of the organic substance, may be separated from these substances by two methods. The whole is either boiled in a platinum capsule with a solution of carbonate of soda, which dissolves the silica, leaving the platinum and the sand, which may then be separated by aqua regia; or the mass is treated at once with aqua regia, when the sand anu silica remain undissolved, and may be separated from each other by boiling with a solution of carbonace of soda. The 
silica dissolved in the solution of carbonate of soda is obtained by supersaturating the solution with muriatic acid, evaporating to dryness, and treating the residue with water; the silica then remains undissolved.

A solution of caustic potash cannot be used for separating the silica from the sand in the analyses of the ashes of vegetable substances, but carbonate of soda must be made use of for this purpose; for the sand is not in most cases pure sand, but usually consists of a mixture of sand and clay, which is either derived from the soil upen which the plants grew, or from the floor of the barn upon which the corn was thrashed, and which has been driven into the straw and the grains of seed during the process of thrashing. This clay is readily decomposed by a solution of caustic potash, the alumina being dissolved, and the amount of silica contained in the substance is rendered incorrect by the silica dissolved out of the clay. When carbonate of soda is used, this decomposition of the clay does not occur.

[To be continued.]

II. On the Transformation of Linear Partial Differential Equations with constant Coefficients to Fundamental Forms. By the Rev. S. Earnshaw, M.A.*

THERE are to be met with in books occasional instances 1 of the transformation of equations of the class here proposed to be considered, but I am not aware that the subject has ever been entered upon systematically. A few years ago, when engaged in some speculations on Laplace's equation, I effected the transformation of all partial differential equations, with constant coefficients, of the second order, for two and three independent variables. The method seems applicable to higher orders and any number of variables. The result of my investigations is, that every partial differential equation of the second order, with constant coefficients, can be transformed into one or other of these two forms, when there are two independent variables,

$$
\begin{aligned}
& \frac{d^{2} u}{d x d y}=a u \quad \text {. . . . . . . . . } \\
& \frac{d^{2} u}{d x^{2}}=a \frac{d u}{d y} ： \text {. . . . . . . . . }
\end{aligned}
$$

and into one or other of these two forms, when there are three independent variables,

- Communicated by the Author. 